\title{
Study of the correlation between structural and photoluminescence properties of CdSe thin films deposited by close-spaced vacuum sublimation
}

\author{
Yu.P. Gnatenko a,*, A.S. Opanasyuk ${ }^{\text {b }}$, M.M. Ivashchenko ${ }^{\text {b }}$, \\ P.M. Bukivskij ${ }^{\text {a }}$ I.O. Faryna ${ }^{\mathrm{a}}$ \\ ${ }^{a}$ Institute of Physics of National Academy of Sciences of Ukraine, 03028 Kyiv, Ukraine \\ b Sumy State University, Rymsky-Korsakov Street 2, 4007 Sumy, Ukraine
}

\section{A R T I C L E I N F O}

Keywords:

A1. Crystal structure

A1. X-ray diffraction

A1. Defects

A3. Polycrystalline deposition

B2. Semiconducting II-VI materials

\begin{abstract}
A B S T R A C T
CdSe polycrystalline films were deposited by a close-spaced vacuum sublimation method at different substrate temperatures $\left(T_{s}\right)$ using glass slides as substrates. At $T_{s} \leq 673 \mathrm{~K}$ the films have a structure with strong dispersion of grain size $(d)$ (from 0.1 to $0.3 \mu \mathrm{m}$ ). In this case the layer-by-layer mechanism determines the growth process of the layers. For $T_{s}=873 \mathrm{~K}$ they have a columnar-like structure with a clear growth texture and the average grain size $d=3-4 \mu \mathrm{m}$. The films obtained at $T_{s}>473 \mathrm{~K}$ are n-type and only correspond to a single wurtzite phase. The crystallites are preferentially oriented with the (102) planes parallel to the substrate. At lower temperatures the films are bi-phase. The microstress level in CdSe films obtained at $T_{s}=873 \mathrm{~K}\left(0.5 \times 10^{-3}\right)$ is considerably smaller than for the films deposited at $T_{s}=773 \mathrm{~K}\left(4.0 \times 10^{-3}\right)$. Increase of the value of $T_{\mathrm{s}}$ improves the stoichiometry of CdSe films. Analysis of the low-temperature photoluminescence (PL) spectra let us determine the nature and energy of point and extended defects in the investigated films. It was shown that the films contain $\mathrm{Na}(\mathrm{Li})$ and $\mathrm{P}$ residual impurities. The results of the structural and PL measurements showed that the CdSe polycrystalline films are of fairly good crystal and optical quality for $T_{s}=873 \mathrm{~K}$ and can be suitable for various applications.
\end{abstract}

(c) 2014 Elsevier Ltd. All rights reserved.

\section{Introduction}

At present, CdSe films are intensively studied. They have direct bandgap energy about $1.75 \mathrm{eV}$ at room temperature and high photosensitivity in the visible spectral region. These films are suitable material for elaboration of a number of optoelectronic devices, such as photoelectrochemical solar cells, photo- and gas-detectors, high-

\footnotetext{
* Corresponding author. Tel.: +380 44 5254717; fax: +380 445251589.

E-mail address: yuriygnatenko@ukr.net (Yu.P. Gnatenko).
}

performance thin film transistors, gamma ray detectors, light-emitting diodes, etc. [1-3]. It should be noted that CdSe thin films can also be used as the absorber layers in the top of tandem solar cells. Therefore, these films are considered to be an important material for photovoltaic applications, since they have a high absorption coefficient and nearly optimum bandgap energy which allows to effectively convert the energy of light into electricity.

The physical properties of CdSe are very sensitive to the deposition conditions and to the technique used. CdSe thin films can be obtained by different techniques such as chemical [4], photochemical [5] and electrochemical vapor 
deposition [6,7], electron-beam [8], magnetron [9] and thermal [10-14] evaporation in vacuum, laser ablation [15] and close-spaced vacuum sublimation (CSVS) [16,17]. Hexagonal cadmium selenide nanorods were grown by a solvothermal technique [18]. It was found that the size, shape and strain structures in the nanorods are defined by their growth mechanism. In order to effectively use those thin films in micro- and optoelectronics it is necessary to optimize their optical, structural and electronic properties. The physical properties of the thin films can be affected by the substrate on which it is deposited and depend on the deposition conditions (evaporator and substrate temperatures, the rate of evaporation). It should be noted that the presence of intrinsic (excitonic) luminescence indicates the optical quality of thin films. For optoelectronic applications it is also very important to use cheap substrates. Earlier it was found, that CdSe films deposited on silicon [15], titanium [19,20] and GaAs [21] substrates present intrinsic luminescence, whereas the films deposited on quartz substrates do not show this luminescence [22]. Therefore, it is very important to obtain the CdSe films of high optical quality by their deposition on cheap substrates, such as quartz or glass. Earlier [23-25], it was shown that a low cost CSVS method makes it possible to deposit the stoichiometric films of binary II-VI semiconductors with controllable physical properties using glass as substrate. However, until now, CdSe polycrystalline films deposited by using this method have not been studied enough.

An important factor, which is responsible for the physical properties of thin films is their crystal structure. It is known, that CdSe exists in two crystal modifications: cubic and hexagonal. The structure of cubic modification is of sphalerite type, while the hexagonal modification has wurzite type structure. For CdSe thin films of wurzite modification, the (002) crystal plane is the plane of their preferred orientation. Usually, the films consist of randomly oriented grains with the appearance of the grain boundaries. Increasing the deposition temperature significantly influences the film structure and induces a considerable increase in the mean grain size and decrease in the grain boundary area $[23,26]$. Besides, the extended and point defects in deposited CdSe thin films are randomly distributed on the surface and in the volume of the films. Therefore they have strong effect on the optical and photoelectric properties of thin films and thus considerably affect their application efficiency. The effect of laser treatment on defect levels distribution in chemicallydeposited CdSe thin films has been studied in [27]. In this case using photoluminescence (PL) measurements a drastic reduction of defect density and redistribution of defect states have been observed. Optical constants (the absorption constant and refractive index) of vacuum-evaporated polycrystalline CdSe thin films were determined in [28]. It was shown that these constants are very sensitive to film thickness and substrate temperature. In particular, anomalous variation of refractive index near the band gap was observed which is explained by the volume and surface imperfections.

The low-temperature PL measurement is a powerful method of investigation of various defects both in bulk crystals [29-31] and in polycrystalline thin films [23,32-34]. We should also note, that the excitonic photoluminescence lines are very sensitive to the various defects in the semiconductor materials.

In this work, we study the structural and photoluminescence properties of CdSe films deposited on the glass substrates by means of a CSVS technique. Comparison of the results of structural investigations and the PL properties of CdSe films deposited under different substrate temperatures allowed us to determine the optimal conditions of their deposition and to evaluate the crystal and optical qualities of the investigated thin films.

\section{Experimental details}

CdSe polycrystalline films were deposited by the CSVS method $[16,23,25]$ on the cleaned glass substrates under different growth conditions, namely: the evaporator temperature was $T_{e}=973 \mathrm{~K}$, the substrate temperature $T_{s}$ varied from $373 \mathrm{~K}$ to $873 \mathrm{~K}$, the growth time was $10 \mathrm{~min}$. The stoichiometric powder of CdSe as an initial material (charge) was used. The CSVS method is described in detail in work [23].

Surface morphology of CdSe thin films and their thickness $l$ were investigated by scanning electron microscopy (SEM). In the last case we used the results of the crosssection of the samples. The measurements of other CdSe films parameters such as the average grain size in the layers, phase analysis, texture, pole density, the coherent scattering domain (CSD) size, microdeformation level $(\varepsilon)$ as well as the lattice parameters were estimated using the methods described in our previous work [23]. In particular, average grain size $(d)$ in the layers was estimated by the Jeffries method [35].

The PL spectra were measured using an SDL-1 grating spectrometer $[29,30,36]$. An LGN-404 argon laser was used for excitation by the $488.8 \mathrm{~nm}$ line. The accuracy of the measurements and temperature stabilization by the UTREKS system was $0.01 \mathrm{~K}$. The spectral resolution of the system was about of $0.1 \mathrm{~nm}$.

\section{Results and discussion}

\subsection{Morphology of CdSe films}

SEM investigation of CdSe films is shown in Fig. 1. The films obtained at low substrate temperatures $T_{s} \leq 673 \mathrm{~K}$ (Fig. 1a) have a structure with strong grain size dispersion (d) (from 0.1 to $0.3 \mu \mathrm{m}$ ). In this case the layers thickness $l=4-6 \mu \mathrm{m}$ was obtained. Here the layer-by-layer mechanism determines the layers growth process (Fig. 1d). It should be noted that for $T_{s} \leq 673 \mathrm{~K}$ the increasing of film thickness does not increase the size of crystallites. Increase of the substrate temperature leads to the change of the growth mechanism. At $T_{s}>673 \mathrm{~K}$ the films have a columnar-like structure with the clear growth texture (Fig. 1 b,c and e,f). The diameter $d$ of columnar-like crystallites strongly depends on the growth conditions $\left(T_{s}, T_{e}, \Delta T=T_{e}-T_{s}\right)$. In particular, the value $d$ increases with thickness $l$ of the films and the substrate temperature. For $T_{e}=973 \mathrm{~K}$ and $T_{s}=873 \mathrm{~K} \mathrm{CdSe}$ films with average 

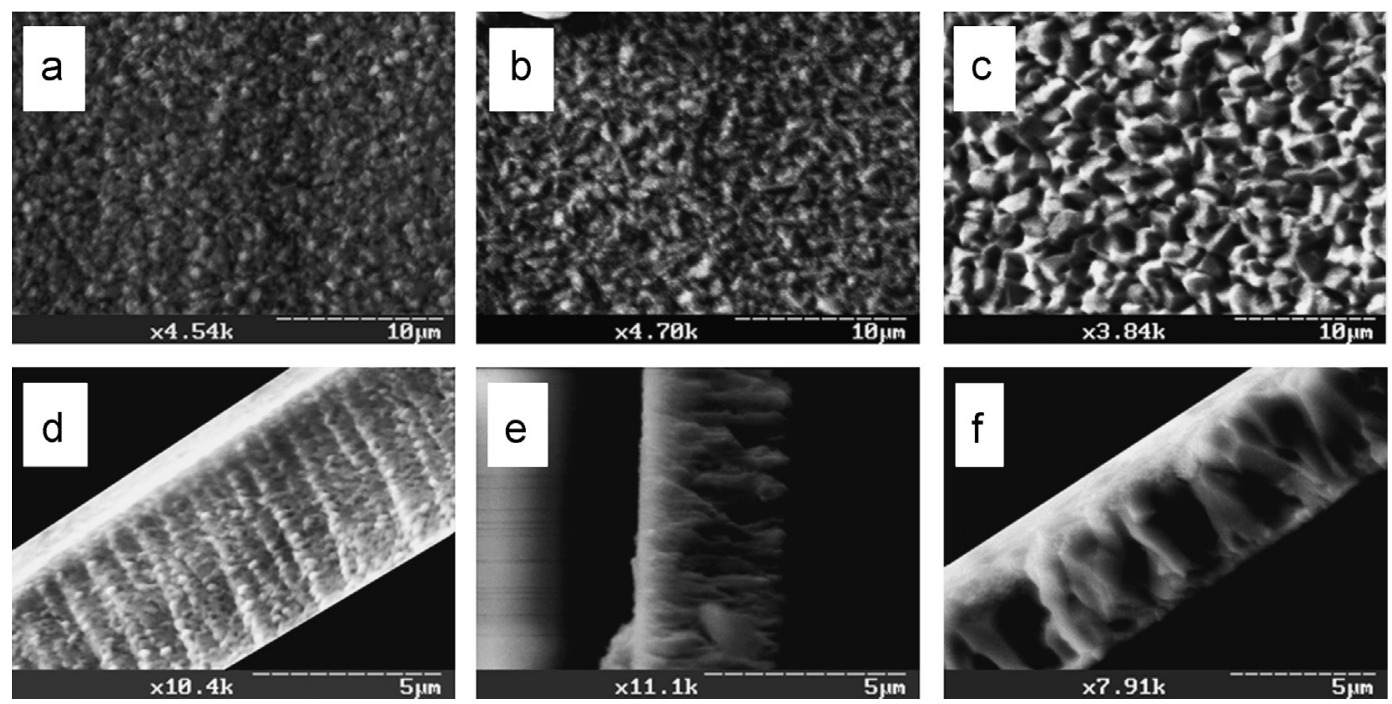

Fig. 1. SEM images of CdSe films surface obtained at the different substrate temperatures: $T_{s}=473 \mathrm{~K}$ (a); $T_{s}=773 \mathrm{~K}$ (b); $T_{s}=873 \mathrm{~K}$ (c) and their cross sections: $T_{s}=473 \mathrm{~K}(\mathrm{~d}) ; T_{s}=773 \mathrm{~K}(\mathrm{e}) ; T_{s}=873 \mathrm{~K}(\mathrm{f})$.

grain size $d=3-4 \mu \mathrm{m}$ and the layers thickness $l=5-6 \mu \mathrm{m}$ were obtained.

\subsection{Phase composition and texture of CdSe films}

X-ray patterns of CdSe films deposited under different growth conditions are presented in Fig. 2. They usually show the peaks from (100), (002), (101), (110), (103), (112), (203), (105), (300), (213) planes of the wurtzite phase. This indicates that the films obtained at $T_{s}>473 \mathrm{~K}$ only correspond to a single wurtzite phase. The growth texture and the axes are perpendicular to these planes.

More information on the film texture can be obtained by the determination of the pole density. It should be noted that the pole density $P_{h k l}$ is a measure of the relative volume of grains belonging to a given [ $h k l]$-crystal direction compared to the respective volume in case of random crystalline distribution [37]. For randomly oriented films the pole density is unity. In the case of the films where this value is more than unity the volume fraction of grains with the given $[h k l]$-crystal direction increases. The films for which the pole density $P_{h k l}$ are larger than unity for several $[h k l]$-crystal directions have multicomponent texture. At the same time, if there is only one [ $h k l]$-crystal direction for which $P_{h k l}>1$, then the film can be considered as having a one-component texture. In this case the film is highly textured.

The results of the pole density calculations for the investigated CdSe films are shown in Fig. 3. They indicate that the value $P_{h k l}$ is much smaller than unity for [110]crystal direction and weakly depends on $T_{s}$ up to $873 \mathrm{~K}$. In the case of [112]-crystal direction the $P_{h k l}$ value decreases from 1 to 0.3 for $T_{s}$ equal to $273 \mathrm{~K}$ and $873 \mathrm{~K}$, respectively. For [002]-crystal direction the pole density is more than unity, which is typical of the films of wurtzite type and often observed in thin films deposited by a thermal evaporation method $[12-14,16]$. It should be noted that the value $P_{002}$ is non-monotonously changing from 3 to 0.7

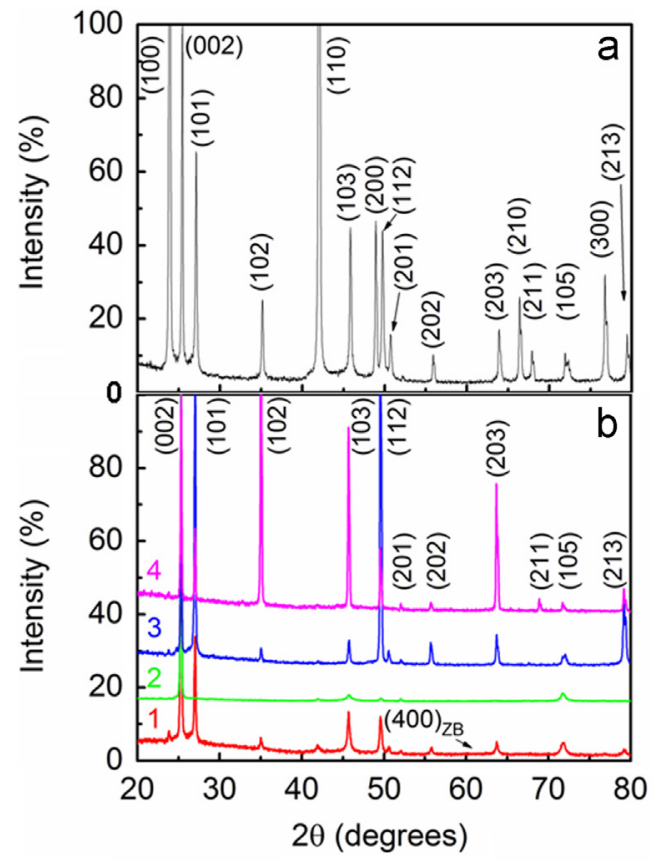

Fig. 2. X-ray patterns of CdSe films deposited at the different substrate temperatures: charge (a); (b): $T_{s}=373 \mathrm{~K}(2) ; T_{s}=573 \mathrm{~K}(3) ; T_{s}=773 \mathrm{~K}(4)$; $T_{s}=873 \mathrm{~K}(5)$.

for the films obtained at $T_{s}=273 \mathrm{~K}$ and $T_{s}=873 \mathrm{~K}$, respectively. However, the pole density $P_{102}$ is strongly increased up to 4 for $T_{s}>800 \mathrm{~K}$. Thus, in this case the crystallites are preferentially oriented with the (102) planes parallel to the substrate. For CdSe films deposited by thermal evaporation the growth textures with [110]- and [112]-crystal directions were observed in [13].

The development of various devices based on semiconductor thin films requires the use of single-phase chalcogenide films with the stable crystalline modification. 


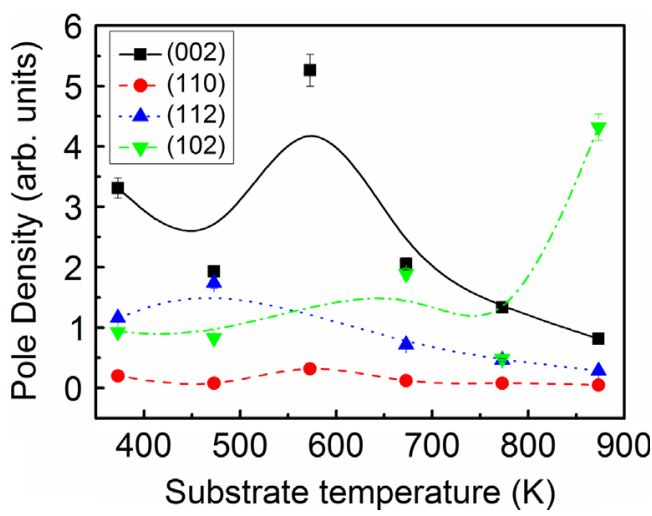

Fig. 3. Pole density $P_{(h k l)}(a)$ as function of substrate temperature.

However, such films can also include a metastable phase that affects their optical and electro-physical characteristics. Usually, CdSe films deposited by the thermal evaporation have the hexagonal structure [11-14,16,17], but bi-phase films have been obtained by this method at $T_{s}=493 \mathrm{~K}[10]$. Detection of metastable cubic phase in CdSe films is a complex task since all the most intense lines for sphalerite and wurtzite phases practically coincide. As can be seen from Fig. 2, (400)-line of cubic phase was observed at the measurement sensitivity limit at $2 \theta \sim 60.90^{\circ}$ for the films obtained at $T_{s}=373 \mathrm{~K}$. Thus, the low-temperature condensates $\left(T_{s}<473 \mathrm{~K}\right)$ are bi-phase, whereas for the high temperature film growth $\left(T_{s}>473 \mathrm{~K}\right)$ there only is a wurtzite phase.

\subsection{Lattice parameters of CdSe films}

The lattice parameter of semiconductors is strongly sensitive to changes in the material characteristics such as stoichiometry, impurity atoms, oxidation, etc. Thus, the measurement of lattice parameter makes it possible to obtain information about these characteristics. Lattice parameters of the charge and CdSe films deposited under different growth conditions were determined using X-ray diffraction patterns line. Analysis showed that the most suitable lines for determining $c$ parameter are (002), (103) and (105) lines, whereas to determine $a$ parameter it is necessary to use (100), (101), (110), (112), (201), (202), (300) and (213) lines.

It should be noted that the experimental values of CdSe lattice parameters of the charge $(a=0.4298 \mathrm{~nm}$, $c=0.7011 \mathrm{~nm}$ ) are in good correlation with data presented in JCPDS [22] for CdSe bulk material $(a=0.4299 \mathrm{~nm}$, $c=0.7010 \mathrm{~nm}$ ). At the same time, lattice parameters of the investigated films, namely, $a=0.4303-0.4316 \mathrm{~nm}$ and $c=0.6998-0.7024 \mathrm{~nm}$, are larger than those for bulk crystal. They are in good agreement with the results $\{a=0.4281-0.4304 \mathrm{~nm} ; c=0.6981-0.7020 \mathrm{~nm}\}$ obtained by Nelson-Riley method for CdSe films deposited by a vacuum evaporation technique [13]. It should be noted that the dependences of the lattice parameters on the substrate temperature are complex which can be explained by changes of material stoichiometry.
Increasing the substrate temperature of CdSe films from $T_{s}=373$ Kto $T_{s}=773 \mathrm{~K}$ induces a significant increase in $a$ parameter from $0.4307 \mathrm{~nm}$ to $0.4316 \mathrm{~nm}$. At the same time, decrease in $c$ parameter from $0.7024 \mathrm{~nm}$ to $0.6998 \mathrm{~nm}$ occurs. This may be caused by lattice deformation as a result of the formation of intrinsic point defects in the films during re-evaporation of selenium, which is more volatile element. As a result of increase of the substrate temperature to $873 \mathrm{~K}$ re-evaporation of cadmium became more efficient improving CdSe films stoichiometry.

\subsection{Microstress and microdomains of the CdSe films}

It should be pointed out that the determination of the substructural characteristics, namely, average CSD size $(L)$ and microstress level $(\varepsilon)$ is based on the measurements of the broadening of (100)-(200), (101)-(202) and (002)(105) diffraction peaks which correspond to a hexagonal phase. Approximation of the diffractions patterns by the Gauss or Cauchy functions allowed us to calculate the values of $L$ and $\varepsilon$. Then, we determined the CSD sizes in the direction parallel to the $c$-axis based on the broadening of (002)-(105) lines\} and perpendicular to the $c$-axis \{based on the broadening of (100)-(200) lines\} for wurtzite phase lattice.

It was established that CSDs have cylindrical form elongated in $c$ direction. In the case of low-temperature condensates $\left(T_{s}=373 \mathrm{~K}\right)$ the height of these cylinders is $L_{(002)} \sim 200 \mathrm{~nm}$ and their diameter is $L_{(100)} \sim 49 \mathrm{~nm}$. For CdSe films, deposited at the conditions close to the thermodynamic equilibrium $\left(T_{s}=873 \mathrm{~K}\right)$, the height of cylinders is $L_{(002)} \sim 62 \mathrm{~nm}$. These changes can be explained by the packing defect formation similarly to ZnS films [26].

It should be pointed out that the microstress level $\varepsilon$ in the films essentially depends on the measured crystal direction. In particular, the value $\varepsilon$ in the direction parallel to the $c$-axis $\left(\varepsilon_{(002)}=(1.7-4.1) \times 10^{-3}\right)$ usually is $2-3$ times larger than in the direction perpendicular to this axis $\left(\varepsilon_{(100)}=(0.5-2.8) \times 10^{-3}\right)$. It should be noted that the microstress level at low substrate temperature increases and then it decreases with increasing $T_{S}$ to $873 \mathrm{~K}$. In particular, the values $\varepsilon$, obtained at $T_{s}=773 \mathrm{~K}$ and $873 \mathrm{~K}$ correspond to $5 \cdot 10^{-3}$ and $0.4 \cdot 10^{-3}$, respectively. Thus, the results of substructure studies for CdSe films presented in this paper indicate that the investigated films are more structurally perfect as compared with the films, obtained by thermal evaporation and hot-wall epitaxy techniques $[10,13,38]$.

\subsection{Photoluminescence of CdSe films}

The low-temperature PL measurements make it possible to determine the nature and energy levels of both point and extended defects as well as the residual impurities in the semiconductor materials [23,24,32,39]. Besides, the PL measurements let us determine the optical quality of the CdSe polycrystalline films. It is very important since the optical quality of semiconductors determines the efficiency of their various applications.

Fig. 4 shows PL spectra of the CdSe polycrystalline films at $4.5 \mathrm{~K}$ for the samples obtained at different substrate 


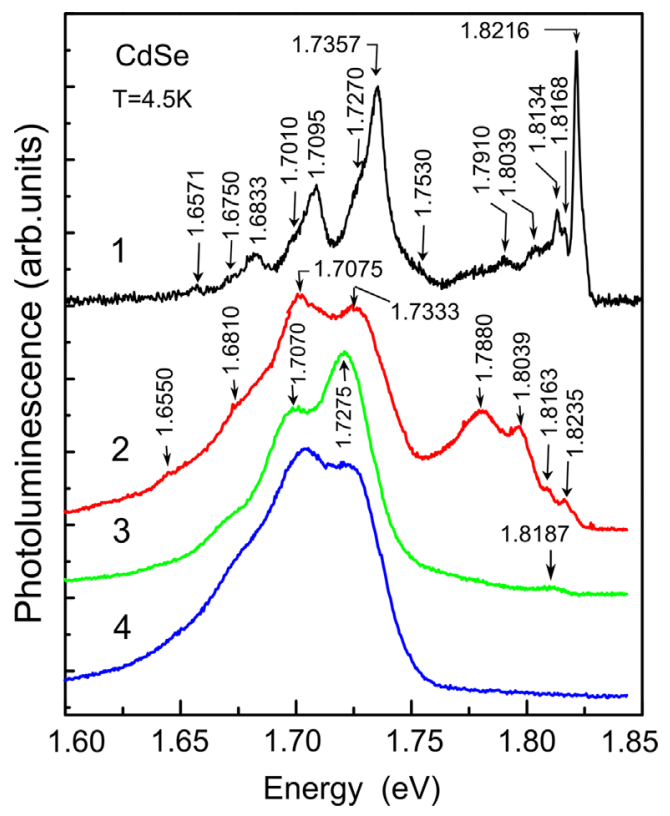

Fig. 4. Low-temperature ( $T=4.5 \mathrm{~K})$ PL spectra for CdSe polycrystalline films grown by the CSVS technique under different technological conditions: $T_{e}=973 \mathrm{~K}$; curves $1-4$ for $T_{s}=873,773,473$ and $373 \mathrm{~K}$, respectively.

temperatures. Curves $1-4$ correspond to the films obtained at $T_{s}$ equal to $893 \mathrm{~K}, 773 \mathrm{~K}, 473 \mathrm{~K}$ and $373 \mathrm{~K}$, respectively. The analysis of the spectrum for the CdSe films obtained at $T=893 \mathrm{~K}$, performed by us in the work [40] showed that the high intensity line observed at $1.7357 \mathrm{eV}$ and other lines at $1.7095 \mathrm{eV}, 1.6833 \mathrm{eV}$ and $1.6571 \mathrm{eV}$ are associated with zero-phonon $I_{\mathrm{DAP} 1}$-line and its 1LO-, 2LO- and 3LOphonon replicas. This emission is caused by recombination of the donor-acceptor pairs (DAP1s) with participation of $\mathrm{Na}$ or Li residual acceptor impurities in CdSe films [41-44]. Taking into account the ionization energies of $\mathrm{Li}(\mathrm{Na})$ acceptors (109 mev) [44] and the shallow donor center (19.0 meV) [45] the distance between DAP1s was found, which corresponds to $R_{1}=6.3 \mathrm{~nm}$.

Other lines which are observed on the long-wavelength wing of the $I_{\mathrm{DAP} 1}$-line and its LO-phonon replicas are caused by the recombination of DAP2s with participation of $P$ residual impurity. Taking into account the energy of the $\mathrm{P}$ acceptor center $\left(E_{V}+0.083 \mathrm{eV}\right)$ and the energy of the donor centers $\left(E_{c}-0.014 \mathrm{eV}\right)$ we obtain for DAP2 the values of $R_{2}$ equal to $15.2 \mathrm{~nm}$. [40]. This means that the concentration of $\mathrm{P}$ atoms in the investigated films is considerably smaller than that of Li or Na atoms. Analysis [40] of the PL spectra of CdSe thin films deposited at different substrate temperatures shows that the concentration of $\mathrm{P}, \mathrm{Na}$ and $\mathrm{Li}$ residual impurities increases with the substrate temperature. This fact may be due to higher diffusion of impurity atoms from the glass substrate at $T_{s}=873 \mathrm{~K}$.

Additional information on the presence and nature of various defects may be obtained from the analysis of the PL spectra in the excitonic region. The high intensity line at $1.8216 \mathrm{eV}$, as shown in [40], is caused by the recombination of donor bound excitons ( $D^{0} X$-line) [42]. The fact that we are able to observe the sharp intense excitonic line in the PL spectrum indicates that the CdSe films are of high optical quality. Furthermore, the presence of the $\mathrm{D}^{0} \mathrm{X}$-line indicates that the CdSe film, obtained at $T_{S}=873 \mathrm{~K}$ is n-type.

It should be noted that the emission associated with extended defects such as dislocations can also occur in semiconductor polycrystalline films [23,24]. The presence of microstress during the film growth may be due to the formation of dislocations in CdSe films. For $T_{S}=873 \mathrm{~K}$ the structure shows very weak intensity in the spectral region 1.75-1.81 eV. It should be noted that at these energies the lines caused by the dislocations appear in the PL spectra [40]. Since they are weak this indicates that the dislocations density in CdSe film deposited at this temperature is relatively small. For the films obtained at $T_{s}=773 \mathrm{~K}$ (Fig. 4 , curve 2) the intensity of $\mathrm{D}^{0} \mathrm{X}$-line at $1.8235 \mathrm{eV}$ is insignificant since in this case the donor concentration is considerably smaller than the film obtained at $T_{S}=873 \mathrm{~K}$. According to [46] this may be caused by increase of the dislocations density for the film obtained at $T_{s}=773 \mathrm{~K}$. These results also correlate with the microstress and microdeformation measurements for CdSe films. In particular, as been seen above, the microstress level for CdSe films deposited at $T_{s}=773 \mathrm{~K}$ is sufficiently more than for $T_{s}=873 \mathrm{~K}$. This may be caused by an increase in dislocation density. Besides, for the films obtained at $T_{s}<873 \mathrm{~K}$ the packing defect formation occurs [40].

As can be seen from Fig. 4 (curve 3 ) the intensity of $D^{\circ} \mathrm{X}$ - and $A^{\circ} X$-lines for CdSe film deposited at $473 \mathrm{~K}$ is very small. At the same time, the high-intensity broad PL bands are observed at $1.7275 \mathrm{eV}$ and $1.7070 \mathrm{eV}$. The first band corresponds to the ZP I IAP2-line and the other band may be associated with 1LO-phonon replica of the zero-phonon $I_{\mathrm{DAP} 1}$-line. As can be seen from Fig. 4 (curve 4), for CdSe film deposited at $373 \mathrm{~K}$ the energy of these lines is the same. The absence of exciton emission lines and the broadening of the bands related to the recombination of donor-acceptor pairs indicates significant heterogeneity of films obtained at low temperatures of glass substrate $\left(T_{s} \leq 473 \mathrm{~K}\right)$ since in this case the layer-by-layer mechanism determines the layers growth process. Besides, these films are bi-phase which induces the packing defect formation.

\section{Conclusion}

In this work we studied the effect of condensation temperature on structural, substructural and photoluminescence properties of CdSe polycrystalline thin films obtained by the CSVS technique. It was shown that the films obtained at low substrate temperatures $T_{s} \leq 673 \mathrm{~K}$ have a structure with strong dispersion of the grain size $(d)$ (from 0.1 to $0.3 \mu \mathrm{m}$ ). In this case the layers thickness $l=$ 4-6 $\mu \mathrm{m}$ was obtained. For higher temperatures they have a columnar-like structure with clear growth texture and the average grain size $d=3-4 \mu \mathrm{m}$ and layers thickness $l=$ 5-6 $\mu \mathrm{m}$. The films obtained at $T_{s}>473 \mathrm{~K}$ only correspond to the single wurtzite phase, while at lower substrate temperatures they are bi-phase. At $T_{s}>800 \mathrm{~K}$ the crystallites are predominantly oriented with (102) parallel to the 
substrate. Increase of the value of $T_{s}$ improves the stoichiometry of CdSe films. Analysis of the low-temperature PL spectra indicates that CdSe films contain $\mathrm{Na}(\mathrm{Li})$ and $\mathrm{P}$ residual impurities. Their concentrations increase with increasing substrate temperature. The presence of excitonic emission indicates the high optical quality of the investigated films at $T_{s}=873 \mathrm{~K}$. These results correlate with the strong decrease of the microstress level in CdSe thin films obtained at $T_{s}=873 \mathrm{~K}\left(0.5 \times 10^{-3}\right)$ in comparison with the films deposited at $T_{s}=773 \mathrm{~K}\left(4.0 \times 10^{-3}\right)$. As a result of these investigations the optimal temperature conditions $\left(T_{e}=973 \mathrm{~K}\right.$ and $\left.T_{s}=873 \mathrm{~K}\right)$ of the growth of polycrystalline CdSe films, which have a single wurtzite phase, were determined. Our findings allowed to considerably improve the structural and optical properties of polycrystalline CdSe films deposited on a low-cost glass substrate which is transparent for CdSe intrinsic emission. These films are of high crystal and optical quality and may be suitable for optoelectronic and photovoltaic applications.

\section{Acknowledgments}

This research has been supported by the Ministry of Education and Science of Ukraine (Grant no. 0110U001151) and by the National Academy of Sciences of Ukraine (Grants nos. BC-157-15 and B-146-15).

\section{References}

[1] P. Mahawela, G. Sivaraman, S. Jeedigunta, J. Gaduputi, M. Ramalingam, S. Subramanian, S. Vakkalanka, C.S. Ferekides, D. L. Morel, Mater. Sci. Eng. B 116 (2005) 283.

[2] S. Kasap, P. Capper, The Springer Handbook of Electronic and Photonic Materials, Springer, Berlin, 2007.

[3] M. Ohring, The Materials Science of Thin Films, Academic press, New York, 1992.

[4] F. Cerdeira, I. Torriani, P. Motisuke, V. Lemos, F. Decker, Appl. Phys. A 46 (1988) (1988) 107

[5] M. Ichimura, K. Takeuchi, A. Nakamura, E. Arai, Thin Solid Films 384 (2001) 157.

[6] A.V. Kokate, U.B. Suryavanshi, C.H. Bhosale, Sol. Energy 80 (2006) 156.

[7] V. Swaminathan, V. Subramanian, K.R. Murali, Thin Solid Films 359 (2000) 113.

[8] N.J.S. Kissinger, M. Jayachandran, K. Perumal, C.S. Raja, Bull. Mater Sci. 30 (2007) 547.

[9] S.K. Bera, S. Chaudhuri, R.P. Gupta, A.K. Pal, Thin Solid Films 382 (2001) 86.

[10] S. Antohe, V. Ruxandra, H. Alexandru, Cryst. Res. Technol. 237-239 (2002) 1559

[11] L. Ion, V.A. Antohe, S Antohe, J. Optoel. Adv.. Mater. 7 (2005) 1847.

[12] E. Bacaksiz, B.M. Basol, M. Altunbas, S. Yilmaz, M. Tomakin, B. Abay, Phys. Status Solidi (a) 244 (2007) 497.

[13] K. Sarman, R. Sarma, H.L. Das, Chalcogenide. Lett. 5 (2008) 153.
[14] R.D. Patel, M.S. Jani, V.M. Pathak, R. Srivastana, Chalcogenide. Lett. 6 (2009) 279

[15] G. Perna, V. Capozzi, M. Ambrico, J. Appl. Phys. 83 (1998) 3337.

[16] C. Baban, G.I. Rusu, P. Prepelita, J. Optoel. Adv. Mater. 7 (2005) 817.

[17] C. Baban, M. Caraman, G.I. Rusu, J. Optoel. Adv. Mater. 8 (2006) 917

[18] U. Pal, P. Santiago, J. Chavez, J.A. Ascencio, J.Nanosci. Nanotechnol. 5 (2005) 609

[19] R. Jäger-Waldau, N. Stücheli, M. Braun, M. Lux Steiner, E. Bucher, J. Appl. Phys. 65 (1988) 2601.

[20] R.P. Siberstein, M. Tomkiewicz, J. Appl. Phys. 54 (1983) 5428.

[21] M. Ambrico, V. Stagno, D. Smaldone, R. Martino, G. Perna, V. Capozz, Proc. SPIE 3404 (1988) 39.

[22] G. Perna, S. Pagliara, V. Capozzi, M. Ambrico, T. Ligonzo, Thin Solid Films 349 (1999) 220.

[23] V. Kosyak, A. Opanasyuk, P.M. Bukivskij, Yu.P. Gnatenko, J. Cryst. Growth 312 (2010) 1726.

[24] Yu.P. Gnatenko, P.M. Bukivskij, A.S. Opanasyuk, D.I. Kurbatov, M.V. Kolesnyk, V.V. Kosyak, H. Khlyap, J. Lumin. 132 (2012) 2885.

[25] D.I. Kurbatov, V.V. Kosyak, M.M. Kolesnyk, A.S. Opanasyuk, S. N. Danilchenko, Yu.P. Gnatenko, Mater. Chem. Phys. 138 (2013) 731.

[26] D. Kurbatov, A. Opanasyuk, H. Khlyap, Phys. Status Solidi A 206 (2009) 1549.

[27] U. Pal, S. Muñoz-Avila, L. Prado-González, R. Silva-González, J.M. Gracia-Jiménez, Thin Solid Films 381 (2001) 155.

[28] U. Pal, D. Samanta, S. Ghorai, A.K. Chaudhuri, J. Appl. Phys. 74 (1993) 6368.

[29] Yu.P. Gnatenko, R.V. Gamernyk, I.A. Farina, P.I. Babii, Phys. Solid State 40 (1998) 1107.

[30] J.A. Garcia, A. Remon, V. Munoz, R. Triboulet, J. Mater. Res. 15 (2000) 1612.

[31] Yu.P. Gnatenko, O.A. Shigil'chev, E. Rutkovski, G. Contreras-Puente, M. Cardenas-Garcia, Phys. Solid State 40 (1998) 564

[32] Yu.P. Gnatenko, P.M. Bukivskij, Y.u.P. Piryatinski, I.O. Faryna, O A. Shigiltchoff, R.V. Gamernyk, Appl. Phys. Lett. 95 (2009) 112109.

[33] Yu.P. Gnatenko, M.S. Brodyn, I.O. Faryna, P.M. Bukivskij, O A. Shigiltchoff, M.S. Furyer, R.V. Gamernyk, N. Kukhtarev, T. Kukhtareva, Phys. Status Solidi 204 (2007) 2431.

[34] M.S. Furyer, P.A. Skubenko, P.M. Bukivskij, L.M. Tarakhan, E.D. Chesnakov, I.G. Vertygel, A.I. Ovcharenko, L.S. Ivanova, R.V. Gamernyk, Yu.P. Gnatenko, J. Appl. Phys. 108 (2010) 103711.

[35] G.F. Vander Voort, Metallography Principles and Practice, ASM International, New York, 1999, 445.

[36] Yu.P. Gnatenko, Yu.P. Piryatinski, R.V. Gamernyk, I.O. Faryna, P.M. Bukivskij, S.Yu. Paranchych, L.D. Paranchych, Proc. SPIE 5209 (2003) 156

[37] M. Adamik, P.R. Barna, I. Tomov, Thin Solid Films 359 (2000) 33.

[38] S. Velumani, X. Mathew, J. Sebastian, S.K. Narayandass, D. Mangalaraj, J. Mater. Sci. Lett. 22 (2003) 25.

[39] J. Aguilar-Hernandez, M. Cardenas-Garcia, G. Contreras-Puente, J. Vidal-Larramendi, Mater. Sci. Eng. B102 (2003) 203.

[40] Yu.P. Gnatenko, P.M. Bukivskij, I.O. Faryna, A.S. Opanasyuk, M.M. Ivashchenko, J.Lumin. 146 (2014) 174.

[41] A. Fasoli, A. Colli, F. Martelli, S. Pisana, Ping Heng Tan, A.C. Ferrari, Nano Res. 4 (2011) 343.

[42] R. Tenne, H. Mariette, C. Levy-Clement, R. Jager-Waldau, Phys. Rev. B 36 (1987) 1204.

[43] Y. Kokubun, H. Watanabe, M. Wada, Japn. Jo. Appl. Phys. 13 (1974) 1393.

[44] C.H. Henry, K. Nassau, J.W. Shiever, Phys. Rev. B 4 (1971) 2453.

[45] G. Mandel, F.F. Morehead, P.R. Wagner, Phys. Rev. 136 (1964) (1964) A826.

[46] A.V. Bazhenov, Yu.A. Osipjan, E.A. Shteinman, Sov. Phys.-Solid State 13 (1972) 2683. 